$38^{\text {th }}$ AIAA Fluid Dynamics Conference and Exhibit, June 23-26, 2008, Seattle, Washington

\title{
Boundary Layer Receptivity due to Roughness and Freestream Sound for Supersonic Flows over Axisymmetric Cones
}

\author{
P.Balakumar \\ Flow Physics and Control Branch \\ NASA Langley Research Center, Hampton, VA 23681
}

\begin{abstract}
The receptivity of supersonic boundary layers due to the interaction of a twodimensional acoustic wave with a three-dimensional roughness is numerically investigated over a 5-degree sharp tipped straight cone at a free stream Mach number of 3.5 and a high Reynolds number of $10^{6} /$ inch. Both the steady and unsteady solutions are obtained by solving the full Navier-Stokes equations using the fifth-order accurate weighted essentially non-oscillatory (WENO) scheme for spatial discretization and using third-order totalvariation-diminishing (TVD) Runge-Kutta scheme for temporal integration. The simulations are performed with different roughness heights and roughness locations. The steady computations with the roughness reveal that the perturbations induced by the roughness remain very close to the wall and do not penetrate to the outer part of the boundary layer. The effects of the roughness persist for about 10 boundary layer thicknesses downstream of each element. The unsteady simulation shows the generation of unstable three-dimensional first mode disturbances within a short distance from the roughness. However, the amplitudes of the instability waves are very small near the neutral point, on the order of $10^{-4}$ compared to the free stream disturbances. The receptivity coefficients due to the roughness elements located very close to the tip are one order of magnitude smaller than those due to the roughness elements located closer to the neutral point.
\end{abstract}

\section{Introduction}

In this work, we are concerned with the fundamental question of receptivity in supersonic boundary layers over axi-symmetric geometries at zero angles of attack. The stability characteristics of supersonic boundary layers have been thoroughly investigated ${ }^{1-3}$. One of the important findings from these investigations is that the most unstable disturbances in supersonic boundary layers are three-dimensional. The waveangles of the most amplified disturbances are inclined around 60-65 degrees from the inviscid streamlines in a boundary layer with an edge Mach number of 3.5. To excite an instability wave in a supersonic boundary layer the frequency, the spanwise wavenumber and the streamwise wavenumber of the disturbances generated inside the boundary layer by external disturbances should match with those of boundary layer instability waves. The frequencies of the disturbances inside the boundary layer in linear cases are the same as that in the free stream. The question is what mechanisms determine the spatial scales of the disturbances generated inside the boundary layer.

On flat plate supersonic boundary layers the wavenumbers of the acoustic and the instability waves are in the same range. For example, the wave numbers of the acoustic and the instability waves near the neutral point for an oblique wave with a spanwise wavenumber of 0.025 are 0.018 and 0.015 respectively ${ }^{4}$. Hence, in contrast to incompressible flows, the instability waves in supersonic boundary layers can be efficiently generated by the free stream acoustic disturbances by weak modulation to the acoustic waves. Fedorov and Khokhlov ${ }^{5,6,7}$ showed using asymptotic theory that eigen solutions are formed near the leading edge region of a flat plate during the diffraction of the acoustic wave by the growing boundary layer. This diffraction zone is very long on the order of $o\left(\varepsilon^{-2} \lambda\right)$ in the streamwise direction, where $\varepsilon$ is an asymptotically small parameter and $\lambda$ is the wavelength of the free stream acoustic disturbances. In our previous studies ${ }^{4,8}$, the generation and the evolution of three-dimensional

1 of 17

American Institute of Aeronautics and Astronautics 
$38^{\text {th }}$ AIAA Fluid Dynamics Conference and Exhibit, June 23-26, 2008, Seattle, Washington

disturbances induced by slow and fast acoustic waves in a supersonic boundary layer over blunt flat plates and wedges at a free stream Mach number of 3.5 were numerically investigated by solving the full threedimensional Navier-Stokes equations. It was found that instability waves are generated within one wavelength of the acoustic wave from the leading edge. The computed receptivity coefficients based on the first mode pressure fluctuations at the wall near the neutral points are about 1.20 and 0.07 for the slow and the fast acoustic waves respectively.

In supersonic boundary layers over axi-symmetric geometries, the azimuthal wavenumber has to be an integer due to the circular periodicity in the azimuthal direction. These azimuthal wavenumbers for the unstable waves in supersonic boundary layers are large ${ }^{9}$, e.g., on the order of 10 to 30 . When these wavenumbers are converted to wavelengths by dividing the circumferential length by the wavenumber, the wavelengths become very small near the nose and increase gradually in the axial direction. The question is then how do the acoustic disturbances in the free stream with long spanwise wavelengths generate these small wavelengths, or the large wavenumber disturbances, inside the boundary layer over axi-symmetric bodies.

There may be two scenarios. One is that three-dimensional plane acoustic waves can interact with the axi-symmetric geometry and can scatter small scale disturbances inside the boundary layer. We considered this possibility in a previous investigation ${ }^{9}$ of the interaction of plane three-dimensional acoustic waves with the cone. The unsteady simulations showed that the modulation of wavelength and the generation of instability waves first occurred near the leading edge in the plane where the constant acoustic phase lines are perpendicular to the cone axis. The receptivity process is very weak in this case and the receptivity coefficient is about $10^{-3}$ compared to 1.20 in the flat plate case. The other scenario is that plane acoustic waves can interact with spatial discontinuities at the surface such as roughness and can scatter a broad band of disturbances inside the boundary layer. Here, the three-dimensionality is introduced by the roughness elements and the required matching of the frequency the spanwise wave number, and the streamwise wave number of the instability wave is generated by the bilinear interaction between the unsteady acoustic field and the steady three-dimensional field induced by the roughness. This mechanism was first explained by Goldstein ${ }^{10,11}$ for incompressible flows. It was shown that the receptivity due to roughness is more efficient than that due to the leading edge non-parallel effect. Choudhari ${ }^{12-15}$ used a finite Reynolds number approach and computed the local coupling coefficients for a wide range of cases including supersonic boundary layers. In this paper, we investigate this possibility by simulating the interaction of a two-dimensional acoustic field with isolated three-dimensional periodic roughness elements in a supersonic boundary layer.

We consider a supersonic flow over an axi-symmetric cone with small bluntness at zero degrees angle of attack. A schematic diagram of the computational set up is depicted in Fig. 1. Periodic threedimensional roughness elements are placed on the surface of the cone close to the nose region. The interaction of two-dimensional acoustic waves with the cone and the roughness element is numerically simulated to investigate the generation and the evolution of the instability waves inside the boundary layer. We note that, in practice, the tip of the cone tends to be non-smooth due to the manufacturing difficulties and is likely to have an irregular surface pattern. The question is, how does this irregular surface roughness near the tip affect the receptivity process? To investigate this issue we placed the roughness elements very close to the tip $\sim 0.05$ in. and performed the unsteady simulations. There were several relevant transition experiments performed at NASA Langley in the Mach 3.5 Supersonic LowDisturbance tunnel. Boundary-layer transition data on a flat plate and on a cone along with measured free stream noise levels and the power spectral distribution of the free stream noise are presented in Ref. 16. As a first step, the computations are performed for the same conditions as those used in the experiment.

\section{Governing Equations}

2 of 17

American Institute of Aeronautics and Astronautics 
The equations solved are the three-dimensional unsteady compressible Navier-Stokes equations in conservation form in cylindrical coordinates

$$
\begin{gathered}
\frac{\partial}{\partial t} r Q+\frac{\partial r F}{\partial x}+\frac{\partial r G}{\partial r}+\frac{\partial H}{\partial \theta}=S . \\
Q=\left[\begin{array}{l}
\rho \\
\rho u \\
\rho v \\
\rho w \\
\rho E
\end{array}\right]
\end{gathered}
$$

Here $(x, r, \theta)$ are the cylindrical coordinates, $(u, v, w)$ are the velocity components, $\rho$ is the density, and $p$ is the pressure. $E$ is the total energy given by

$$
\begin{aligned}
& E=e+\frac{u^{2}+v^{2}+w^{2}}{2}, \\
& e=c_{v} T, p=\rho R T .
\end{aligned}
$$

Here $e$ is the internal energy and $T$ is the temperature. The fluxes $F, G, H$ and the source term $S$ are described in Ref. 9. The viscosity $(\mu)$ is computed using Sutherland's law and the coefficient of conductivity $(k)$ is given in terms of the Prandtl number $P r$. The variables $\rho, p, T$ and velocity are nondimensionalized by their corresponding reference variables $\rho_{\alpha}, p_{\alpha}, T_{\alpha}$ and $\sqrt{R T_{\infty}}$ respectively. The reference value for length is computed by $\sqrt{v x_{0} / U_{\infty}}$, where $x_{0}$ is a reference location. For the computation, the equations are transformed from the physical coordinate system $(x, r, \theta)$ to the computational curvilinear coordinate system $(\xi, \eta, \zeta)$ in a conservative manner. The corresponding Cartesian coordinates are $(x, y, z)$ with $z$ and $y$-axis oriented along $\theta=0$ and 90 degree lines respectively, Fig. (1).

\section{A. Solution Algorithm}

The governing equations are solved using a 5th order accurate weighted essentially non-oscillatory (WENO) scheme for space discretization and using a third order, total variation diminishing (TVD) Runge-Kutta scheme for time integration. These methods are suitable in flows with discontinuities or high gradient regions. The governing equations are solved discretely over a uniformly spaced, structured computational grid, where flow properties are known point wise at the grid nodes. In a given direction, the spatial derivatives are approximated to a higher order at the nodes, using the neighboring nodal values in that direction. The resulting equations are then integrated in time to get the point values as a function of time. Since the spatial derivatives in one direction are independent of the other coordinate directions, the method is easily extended to multiple spatial dimensions. It is well known that approximating a discontinuous function by a higher order (two or more) polynomial generally introduces oscillatory behavior near the discontinuity, and this oscillation increases with the order of the approximation. The 
$38^{\text {th }}$ AIAA Fluid Dynamics Conference and Exhibit, June 23-26, 2008, Seattle, Washington

essentially non-oscillatory (ENO) and the improvement of this, WENO methods, were developed to maintain the higher order approximations in the smooth regions and to eliminate or suppress the oscillatory behavior near the discontinuities. These are achieved by systematically adopting or selecting the stencils based on the smoothness of the function that is being approximated. Reference 17 explains the WENO and the TVD methods and the formulas. Reference 18 gives the application of the ENO method to the N-S equations. Reference 19 describes in detail the solution method implemented in this computation.

At the outflow boundary, characteristic boundary conditions are used. At the wall, viscous conditions are used for the velocities and a constant temperature condition is employed for the temperature. The density at the wall is computed from the continuity equation. In the azimuthal direction, symmetric and periodic conditions are used at the boundaries. Hence only half the wavelength is considered in the computations. In the mean flow computations, the free-stream values are prescribed at the upper boundary that lies outside the bow shock. In the unsteady computations, the acoustic perturbations are superimposed on the uniform mean flow at the upper boundary. The procedure is to first compute the steady mean flow by performing unsteady computations using a variable time step until the maximum residual reaches a small value $\sim 10^{-11}$. A CFL number of 0.10 is used near the nose region and 0.50 is used downstream. The next step is to introduce unsteady disturbances at the upper boundary of the computational domain and to perform time accurate computations to investigate the interaction and evolution of these disturbances downstream. There exists a singularity along the symmetry axis $r=0$. This singularity is avoided by placing the symmetry axis in the middle of the two grid points around it.

The two-dimensional acoustic field that impinges on the outer boundary is taken to be in the following form.

$$
p_{a c}^{\prime}=\operatorname{Real}\left\{\tilde{p}_{a c} e^{i \alpha_{a c} x-i \omega t}\right\}
$$

Here $\alpha_{a c}$ is the acoustic wavenumber, and $\omega$ is the frequency of the acoustic disturbance.

\section{Results}

The Computations are performed for supersonic flows over a 5-degree half-angle cone with a small blunt tip. Table 1 gives the flow parameters and Fig. 1 shows the schematic diagram of the computational set up.

\section{Table 1 Flow parameters for the wind tunnel model}

Free stream Mach number: $M_{\alpha}=3.5$

Free stream Reynolds number: $R e_{\alpha}=1.0 * 10^{6} /$ in.

Free stream density: $\rho_{\alpha}=2.249 * 10^{-2} \mathrm{lbm} / \mathrm{ft}^{3}$

Free stream pressure: $p_{\alpha}=187.74 \mathrm{lbf} / \mathrm{ft}^{2}$

Free stream velocity: $U_{\alpha}=2145.89 \mathrm{ft} / \mathrm{s}$

Free stream temperature: $T_{\alpha}=156.42{ }^{\circ} \mathrm{R}$

Free stream kinematic viscosity: $v_{\infty}=1.7882 * 10^{-4} \mathrm{ft}^{2} / \mathrm{s}$

Wall temperature: $T_{\alpha}=476.0^{\circ} \mathrm{R}$

Prandtl number: $P r=0.72$

Ratio of specific heats: $\gamma=1.4$ 
Length scale $\sqrt{\frac{v_{\infty} x_{0}}{U_{\infty}}}=5.892 * 10^{-5} f t .\left(x_{0}=0.5\right.$ in. $)$

Local Reynolds number: $\sqrt{\operatorname{Re}_{x}}=\sqrt{\frac{U_{e} x}{v}}$

The boundary layer thickness at $x_{0}=0.5$ in.: $\delta_{0}=.0055$ in.

Non-dimensional frequency $F=1 * 10^{-5}$ is equivalent to $41.0 \mathrm{kHz}$

The non-dimensional frequency $F$ is defined as $F=\frac{2 \pi v_{\infty} f}{U_{\infty}^{2}}$,

where $f$ is the frequency in Hertz.

The nose region of the cone is modeled as a circle of the form

$$
\left(x-r_{0}\right)^{2}+y^{2}=r_{0}^{2}
$$

Here $r_{0}$ is the radius of the blunt nose tip. The circular nose is smoothly merged with the cone by a tenth order polynomial. Simulations are performed for a nose radius of $r_{0}=0.001$ inches at a unit Reynolds number of $1.0 * 10^{6} /$ inch.

The grid stretches in the $\eta$ direction close to the wall and is uniform outside the boundary layer. In the $\xi$ direction, the grid is symmetric about the tip and very fine near the nose and is uniform in the flat region. The grid is uniform in the azimuthal direction. The outer boundary outside of the shock follows a parabola with its vertex located a short distance upstream of the nose to capture the boundary layer accurately. The computational domain extends from $x=-0.015$ to 8.0 inches in the axial direction. The grid distribution in the axial and in the azimuthal directions over the roughness is shown in Fig. 2. Calculations were performed using grid sizes of $(2001 * 251 * 11)$ and $(3001 * 251 * 25)$ depending on the roughness height. Due to the very fine grid requirement near the nose, the allowable time step is very small and it becomes very expensive to simulate the unsteady computations in the entire domain at once. To overcome this, calculations are performed in two steps. First, the computations are done near the nose region with a very small time step. Second, the flow properties in the middle of this domain are fed as inflow conditions for the second larger domain wherein a larger time step can be used.

\section{A. Linear instability}

The linear stability results for the axi-symmetric similarity boundary layers over the 5-degree half angle cone at a free stream Mach number of 3.5 were presented earlier in Ref. 9. The conditions downstream of the shock on the surface of the cone are:

$$
\begin{array}{ll}
\mathrm{M}_{\mathrm{s}} & =3.355 \\
\mathrm{~T}_{\mathrm{s}} & =1.06 \mathrm{~T}_{\infty} \\
\rho_{\mathrm{s}} & =1.15 \rho_{\infty} \\
\mathrm{p}_{\mathrm{s}} & =1.16 \mathrm{p}_{\infty} \\
\mathrm{Re}_{\mathrm{s}} / \mathrm{in} & =1.075^{*} 10^{6}
\end{array}
$$

Here, $M_{s}, T_{s}, \rho_{s}$ and $p_{s}$ are the inviscid surface Mach number, temperature, density, and the pressure respectively. The most amplified frequency is about $F=2.0-2.5^{*} 10^{-5}$ and the azimuthal wave number of the most amplified wave is about $m=20 \sim 25$. Figures 3(a) and (b) depict the streamwise wave number, growth rate, phase speed and the wave angle for the most amplified disturbance with $m=20$ and $F=$ 
$2.0 * 10^{-5}$. The wave number increases from 0.008 to 0.085 when the Reynolds number $\sqrt{ } \mathrm{Re}_{\mathrm{x}}$ increases from 410 to 3000 . The streamwise wave number near the neutral point is about 0.021 . The dimensional wavelengths of the instability waves are $0.290,0.214$ and 0.207 inches at $\sqrt{ } \mathrm{Re}_{\mathrm{x}}=410,1000$ and 3000 respectively and the wavelength of the two-dimensional acoustic wave is about 0.224 inches. It is also noticed that the phase speed is approaching 1.0 close to $\sqrt{ } \mathrm{Re}_{\mathrm{x}}=410$. This suggests that the instability mode for these parameters merges with the continuous spectrum for the vorticity mode near this Reynolds number. The phase speed decreases to about 0.66 around $\sqrt{ } \mathrm{Re}_{\mathrm{x}}=1870$ and then increases to about 0.71 at $\sqrt{ } \mathrm{Re}_{\mathrm{x}}=3000$. The phase angle decreases from 89 degrees to 42 degrees within this Reynolds number range. The Mach angle is about 72.6 degrees for the Mach number of $M=3.5$. Hence, below a Reynolds number of $\sqrt{ } \mathrm{Re}_{\mathrm{x}}=1550$ the wave direction is outside of the Mach region. This may have implications in the receptivity process in the supersonic flows over axisymmetric geometries. In the experiment ${ }^{16}$ the transition occurred close to a Reynolds number of 3000 and this yields an N-Factor of 8.5 for a constant $m$.

\section{B. Roughness}

A three dimensional, azimuthally periodic roughness strip is placed on the surface of the cone close to the nose region. The shape of the roughness is in the form

$$
y_{c}(x, \theta)=h e^{-\sigma\left(\frac{x-x_{r}}{l}\right)^{2}} \cos m \theta
$$

Here $y_{c}$ is the height of the roughness normal to the surface of the cone, $h$ is the maximum height, $x_{r}$ is the axial location of the roughness, $m$ is the azimuthal wavenumber, $l$ is the length scale and $\sigma$ is a constant that determines the width of the roughness in the axial direction. The Fourier transform of the roughness shape is given by

$$
\begin{aligned}
\tilde{y}_{c}\left(k_{x}\right) & =\frac{1}{2 \pi} \int_{-\infty}^{\infty} y_{c}(x) e^{-i k_{x} x} d x \\
& =\bar{h} \frac{1}{2 \sqrt{\pi \sigma}} e^{-\frac{k_{x}^{2}}{4 \sigma}}
\end{aligned}
$$

Here the variables $k_{x}$ and $h$ are non-dimensionalised by the length scale $l$. It is seen that for small $k_{x}$ values the spectrum for this roughness shape is almost flat. Computations are performed for $m=20$ and for different $x_{r}, h$ and $\sigma$ that are given in Table 2 .

Table 2 Location and the height of the roughness

\begin{tabular}{|c|c|c|c|}
\hline $\mathbf{X}_{\mathbf{r}}$ (in.) & h (in.) & $\boldsymbol{\sigma}$ & $\mathbf{h} / \mathbf{\delta}$ \\
\hline $\mathbf{0 . 5 0}$ & $\mathbf{0 . 0 0 0 7}$ & $\mathbf{0 . 0 1}$ & $\mathbf{1} / \mathbf{8}$ \\
\hline $\mathbf{0 . 2 5}$ & $\mathbf{0 . 0 0 0 5}$ & $\mathbf{0 . 0 1}$ & $\mathbf{1} / \mathbf{8}$ \\
\hline 0.25 & $\mathbf{0 . 0 0 1 0}$ & $\mathbf{0 . 0 1}$ & $\mathbf{1} / \mathbf{4}$ \\
\hline 0.05 & $\mathbf{0 . 0 0 0 2}$ & $\mathbf{0 . 0 2}$ & $\mathbf{1 / 8}$ \\
\hline $\mathbf{0 . 0 5}$ & $\mathbf{0 . 0 0 0 5}$ & $\mathbf{0 . 0 2}$ & $\mathbf{1} / \mathbf{3}$ \\
\hline 0.05 & $\mathbf{0 . 0 0 1}$ & $\mathbf{0 . 0 2}$ & $\mathbf{2} / \mathbf{3}$ \\
\hline
\end{tabular}

The local Reynolds numbers at these axial locations $x_{r}=0.05,0.25$ and 0.50 inches are about $\sqrt{\mathrm{Re}_{\mathrm{x}}}=$ 230,520 and 730 respectively. The density profiles without the roughness elements at the axial locations where the roughness elements are located are plotted in Figs. 4(a)-(f). The shapes of the roughness 
$38^{\text {th }}$ AIAA Fluid Dynamics Conference and Exhibit, June 23-26, 2008, Seattle, Washington

profiles are also depicted in the figures for comparison. The roughness height compared to the boundary layer thickness is about $1 / 8$ at $x_{r}=0.05$ with the roughness height of 0.0002 inches and is $1 / 8$ at $x_{r}=0.25$ and 0.50 inches with roughness heights of 0.0005 and 0.0007 inches.

\section{Mean flow with and without roughness}

The mean flow density contours and boundary layer profiles computed using the WENO code without the roughness elements are given in Ref. 9. The computations are performed at a unit Reynolds number of $1.0 * 10^{6} /$ inch which yields a Reynolds number based on the nose radius of $10^{3}$. Figure 5 (a) shows the density contours in a larger domain while figure 5(b) shows the flow field near the nose region. The leading edge shocks are located approximately at 0.0002 inches upstream of the leading edge. The boundary layer profiles approach the similarity profiles at about $x=1.0$ inches from the leading edge.

Figures 6(a-d) show contours of the radial velocity component, $v$, close to the roughness element for different cases. The figure reveals the modification due to the roughness and the radiation of the Mach waves that originate from the roughness. The perturbations and the strength of the Mach waves increase with the roughness height. In the next three figures we present the flow fields near the roughness elements in different planes. Figures 7(a-b) show the contours of the azimuthal velocity, $w$, component above the roughness element in the $(x, r)$ plane. Figures 8(a-d) depict the contours of the $w$ velocity in the cross sectional $(y, z)$ planes across the roughness for different cases. Figures $9(\mathrm{a}-\mathrm{b})$ show the contours of the $w$ velocity in the plan view $(x, r \theta)$ plane. The figure shows the lifting of the fluid away from the roughness in upstream of the roughness and turning back towards the wall in downstream of the roughness similar to formation of a longitudinal vortex. However, the strength diminishes in a short distance downstream of the roughness. In figure 10 we plotted the density and the azimuthal velocity profiles above the roughness. Figure 10(a) shows the results for the case $x_{r}=0.05$ and $h=0.0005$ inches and figure 10(b) shows the results for the cases $x_{r}=0.25$ and $h=0.0005,0.001$ inches, and $x_{r}=0.50$ and $h=0.0007$ inches. In figure 10(b) we also plotted the eigenfunction profile for the $w$ velocity component obtained from the linear stability computations for the case with $F=2.0^{*} 10^{-5}$ and $m=20$. One important observation is that the steady perturbations induced by the roughness are confined close to the surface and do not penetrate to the outer part of the boundary layer where the eigenfunctions peak in supersonic boundary layers. Another observation is that the amplitude of the perturbation decays to small values near the region where the eigenfunction peaks. The maximum amplitude of the perturbations is about 0.03 and it reduces to 0.0015 near the region where the eigenfunction peaks.

\section{Interaction of a two-dimensional acoustic wave with the cone without the roughness}

The governing equations and the solutions for plane acoustic waves are given in Ref. 4. After the mean flow is computed, two-dimensional plane acoustic disturbances are introduced at the outer boundaries and the time accurate simulations are performed to investigate the evolution of disturbances inside the boundary layer. The simulations are performed for a non-dimensional frequency of $F=2.00^{*} 10^{-}$ 5 . The amplitude of the free stream acoustic disturbances is $p_{a c} / p_{\infty}=1.0 * 10^{-5}$. Figures 11(a-b) show the evolution of the unsteady perturbations obtained from the simulations. Figure 11(a) shows the contours of the density fluctuations in the entire domain and Fig. 11(b) shows the instantaneous pressure fluctuations along the wall. As expected the two-dimensional disturbances are almost neutral and the amplitude increased very slowly by a factor of 3.0 from $x=0.05$ to 8.0 inches. Figure 12 shows the amplitude of the density fluctuations across the boundary layer at different axial locations $x=0.05,0.25$ and 0.50 inches where the roughness elements were later located. It is seen that the amplitudes of the fluctuations inside the boundary layer have the same magnitudes as in the free stream and the amplitudes peak near the outer part of the boundary layer. If we compare figures 10 (b) and 12 at $x=0.25$ and 0.50 we see that the steady perturbations peak at about $y / \delta \sim 1 / 8$, the density perturbations due to the acoustic wave without the roughness peaks at $y / \delta \sim 2 / 3$ and the eigenfunction for the three-dimensional instability waves peaks at $y / \delta \sim 3 / 5$. As we discussed earlier the instability waves are going to be generated by the bilinear interaction

7 of 17

American Institute of Aeronautics and Astronautics 
between the flow fields shown in figures 10 and 12 . Hence we expect the interaction will be weak in generating the instability waves.

\section{E. Interaction of a two-dimensional acoustic wave with the cone with the roughness.}

After the mean flow is computed for the cone with the roughness element, two-dimensional plane acoustic disturbances are introduced at the outer boundaries as discussed in the previous section. The simulations are performed for a non-dimensional frequency of $F=2.00 * 10^{-5}$ and for an azimuthal wavenumber of $m=20$ with different roughness locations and heights. To remain in the linear regime, very small initial amplitude of $\tilde{p}_{a c} / p_{\infty}=1.0 * 10^{-5}$ is prescribed for the free-stream acoustic waves. Figure 13 shows the results for the evolution of the unsteady fluctuations obtained from the simulation at a fixed time for the case $x_{r}=0.50$ and $h=0.0007$ inches. Figure 13(a) shows the contours of the density fluctuations near the roughness in the 0-degrees phase plane and Fig. 13(b) depicts the contours for the azimuthal velocity, $w$, inside the boundary layer in the 90 -degrees phase plane. It should be noted that the azimuthal velocity field shown in Fig. 13(b) is purely generated by the interaction of a two-dimensional acoustic wave with the three-dimensional roughness elements. The scattering of the unsteady field by the roughness along the Mach lines and inside the boundary layer and the generation of the disturbances downstream of the roughness are clearly seen in the figure 13(b). Figures 14(a-c) show the instantaneous three-dimensional pressure fluctuations along the wall near the roughness for the cases $\left(x_{r}, h\right)=(0.50$, $0.0007),(0.25,0.0005)$ and $(0.25,0.001)$. Similarly, figure 15 shows the corresponding results farther downstream from the roughness array. Figures 16(a-b) show the amplitude of the pressure fluctuations along the wall and the maximum three-dimensional density fluctuations in a log scale for the cases $\left(x_{r}, h\right)$ $=(0.50,0.0007)$ and $(0.25,0.0005)$. Figure 16 also includes the results from the parabolized stability equations (PSE) computations obtained for the same mean boundary layer profiles. The figures clearly show the initial generation and the eventual exponential growth of the instability waves inside the boundary layer. However, in all the cases the amplitudes of the fluctuations generated by this process are very small. The pressure fluctuations close to the roughness elements are on the order of $10^{-8}$ and the maximum density fluctuations are on the order of $10^{-6}$.

The growth of the disturbances agrees very well with the PSE results downstream of the neutral point. Following the PSE results up to the neutral point, the initial amplitude of the instability waves at the neutral point can be estimated. From these values the receptivity coefficients defined by the initial amplitude of the pressure fluctuations at the wall at the neutral point non-dimensionalised by the freestream acoustic pressure can be evaluated.

$$
C_{\text {recpt }, p_{\text {wall }}}=\frac{\left(p_{\text {wall }}\right)_{n}}{p_{a c}}
$$

Similarly, the receptivity coefficients based on the maximum density fluctuations inside the boundary layer normalized by $\rho_{a c}$ can be defined. The computed receptivity coefficients for different cases are given in Table 3.

$\underline{\text { Table } 3 \text { Receptivity coefficients for different roughness locations and heights }}$

\begin{tabular}{|c|c|c|c|}
\hline $\mathbf{X}_{\mathrm{r}}$ (in.) & h (in.) & $C_{\text {recpt }, p_{\text {wall }}}$ & $C_{r e c p t, \rho_{\max }}$ \\
\hline 0.50 & 0.0007 & $3.0 * 10^{-4}$ & $3.1 * 10^{-2}$ \\
\hline 0.25 & 0.0005 & $1.2 * 10^{-4}$ & $1.2 * 10^{-2}$ \\
\hline 0.25 & 0.0010 & $1.8 * 10^{-4}$ & $1.7 * 10^{-2}$ \\
\hline 0.05 & 0.0002 & $2.7 * 10^{-6}$ & $2.1 * 10^{-4}$ \\
\hline 0.05 & 0.0005 & $3.3 * 10^{-5}$ & $2.9 * 10^{-3}$ \\
\hline 0.05 & 0.001 & $9.9 * 10^{-5}$ & $9.0 * 10^{-3}$ \\
\hline
\end{tabular}

8 of 17

American Institute of Aeronautics and Astronautics 
As was discussed earlier, the receptivity coefficients are much smaller on the order of $10^{-4}$ compared to 1.20 in the flat plate case. The receptivity is the highest when the roughness is located closer to the neutral point, $x_{r}=0.50$.

Similarly, we performed the simulation with the roughness elements located very close to the tip of the cone, $x_{r}=0.05$ inches. Figures 17 and 18 show the results for these cases $\left(x_{r}, h\right)=(0.05,0.0002),(0.05$, $0.0005)$ and $(0.05,0.001)$. Figure 17 presents the pressure fluctuations along the wall closer to the roughness and figure 18 shows the pressure fluctuations and the maximum density fluctuations in $\log$ scale. The free stream acoustic pressure levels for these cases are $\tilde{p}_{a c} / p_{\infty}=1.0 * 10^{-4}$. We also performed grid refinement studies with a smaller computational domain in the radial direction but with the same number of grid points. The results are included in figures 17(a) and (b). The figures confirm that the results obtained are accurate and independent of grid resolutions. We computed the receptivity coefficients by following the PSE results as was done previously. The receptivity coefficients are given in Table 3. The receptivity coefficients are one order smaller than that was obtained for the earlier cases with the roughness elements located closer to the neutral point. This suggests that roughness elements located closer to the tip do not introduce large disturbances in the supersonic boundary layers over a cone with slightly blunt nose tip.

\section{Summary and Conclusions}

The supersonic boundary layer receptivity process due to the interaction of two-dimensional acoustic disturbances with a three-dimensional element for the flow over a sharp 5-degree cone is numerically investigated at a free stream Mach number of 3.5 and at a high Reynolds number of $10^{6} / \mathrm{inch}$. Both the steady and unsteady solutions with and without roughness elements and unsteady solutions are obtained by solving the full Navier-Stokes equations using the $5^{\text {th }}$-order accurate weighted essentially nonoscillatory (WENO) scheme for space discretization and using third-order total-variation-diminishing (TVD) Runge-Kutta scheme for time integration.

The roughness elements are placed at different axial locations close to the nose region. The roughness height varies between $1 / 8$ to $2 / 3$ of the boundary layer thickness. The mean flow field showed a displacement of the boundary layer and generation of Mach waves near the roughness elements. The effects of the roughness persist for about 10 boundary layer thicknesses downstream of the roughness. One important finding is that the steady perturbations induced by the roughness elements are confined close to the wall, within $1 / 8^{\text {th }}$ of the boundary layer thickness. These disturbances do not penetrate outside the boundary layer and decay very fast in the outer part of the boundary layer. The unsteady simulations without the roughness element showed as expected that the two-dimensional disturbances inside the boundary layer remain almost neutral up to the transition point. The unsteady simulations with the roughness revealed that due to the interaction of the two-dimensional acoustic disturbances with the threedimensional roughness elements, the first mode instability waves are generated within a short distance from the roughness. However, the amplitude of the excited disturbances near the neutral point is very small compared to the free stream acoustic disturbances. The receptivity coefficient based on the pressure fluctuations at the wall near the neutral point is about $5.0^{*} 10^{-4}$ for the case $\left(x_{r}, h\right)=(0.50,0.0007)$. It is also observed that the receptivity coefficient increases when the roughness is located close to the neutral point. The receptivity coefficient is an order of magnitude smaller when the roughness is located very close to the tip of the cone. This implies that the isolated imperfections produced by manufacturing defects should not introduce large disturbances inside the boundary layer. One question that is not investigated is the effect of distributed roughness in the generation of instability waves in supersonic boundary layers. That will be addressed in future work.

9 of 17

American Institute of Aeronautics and Astronautics 


\section{References}

1. Lees, L. and Lin, C. C., "Investigation of the Laminar Boundary Layer in a Compressible Fluid," NACA Technical Note, Vol. 83, Sept. 1946, pp. 115.

2. Lees, L. and Reshotko, E., "Stability of Compressible Boundary Layer," Journal of Fluid Mechanics, Vol. 12, 1962, pp. 455-477.

3. Mack, L. M., "Boundary Layer Stability Theory”, JPL 900-277 Rev. A, Nov. 1969, Jet Propulsion Lab., Pasadena, Calif.

4. P. Balakumar, "Transition in a Supersonic Boundary layer Due to Acoustic Disturbances," AIAA Paper 2005-0096, 2005.

5. Fedorov, A. V., and Khokhlov, A. P., " Excitation of Unstable Modes in a Supersonic Boundary Layer by Acoustic Waves," Fluid Dynamics, No. 9, pp. 456-467, 1991.

6. Fedorov, A. V., and Khokhlov, A. P., "Sensitivity of a Supersonic Boundary Layer to Acoustic Disturbances," Fluid Dynamics, No. 27, pp. 29-34, 1992.

7. Fedorov, A. V., "Receptivity of High Speed Boundary Layer to Acoustic Disturbances," AIAA Paper 20022846, 2002.

8. P. Balakumar, "Stability of a Supersonic Boundary layer Over Blunt Wedges," AIAA Paper 2006-0137, 2006.

9. P. Balakumar, "Receptivity of Supersonic Boundary Layers Due To Acoustic Disturbances Over Blunt Cones ," AIAA Paper 2007-4491, 2007.

10. Goldstein, M. E., "The evolution of Tollmien-Schlichting waves near a leading-edge", J. Fluid Mechanics, Vol. 127, 1983, pp. 59-81.

11. Goldstein, M. E., "Scattering of acoustic waves into Tollmien-Schlichting waves by small streamwise variations in surface geometry", J. Fluid Mechanics, Vol. 154, 1985, pp. 509-529.

12. Choudhari, M. and Streett, C. L., "Boundary Layer Receptivity Phenomena in Three-Dimensional and High-Speed Boundary Layers," AIAA Paper 90-5258, 1990.

13. Choudhari, M. and Streett, C. L., "A Finite Reynolds Number Approach for the Prediction of Boundary Layer Receptivity in Localized Regions," NASA TM 102781, Jan. 1991.

14. Choudhari, M., “ Acoustic Receptivity of Compressible Boundary Layers: Receptivity via SurfaceTemperature Variations," NASA Contractor Report 4599, June 1994.

15. Choudhari, M. and Streett, C. L., "Theoretical Prediction of Boundary-Layer Receptivity," AIAA Paper 942223, 1994.

16. Chen, F. J., Malik, M. R., Beckwith, I. E., "Comparison of Boundary Layer transition on a Cone and Flat Plate at Mach 3.5," AIAA-88-0411, 1988.

17. Shu, Chi-Wang, "Essentially Non-Oscillatory and Weighted Essentially Non-Oscillatory Schemes for Hyperbolic Conservation Laws," NASA/CR-97-206253 and ICASE Report N0. 97-6

18. Atkins, H. L., "High-Order ENO Methods for the Unsteady Compressible Navier-Stokes Equations," AIAA Paper 91-1557, 1991.

19. Balakumar, P., Zhao, H., and Atkins, H., "Stability of Hypersonic Boundary_layers Over a Compression Corner," AIAA Paper 2002-2848, 2002. 


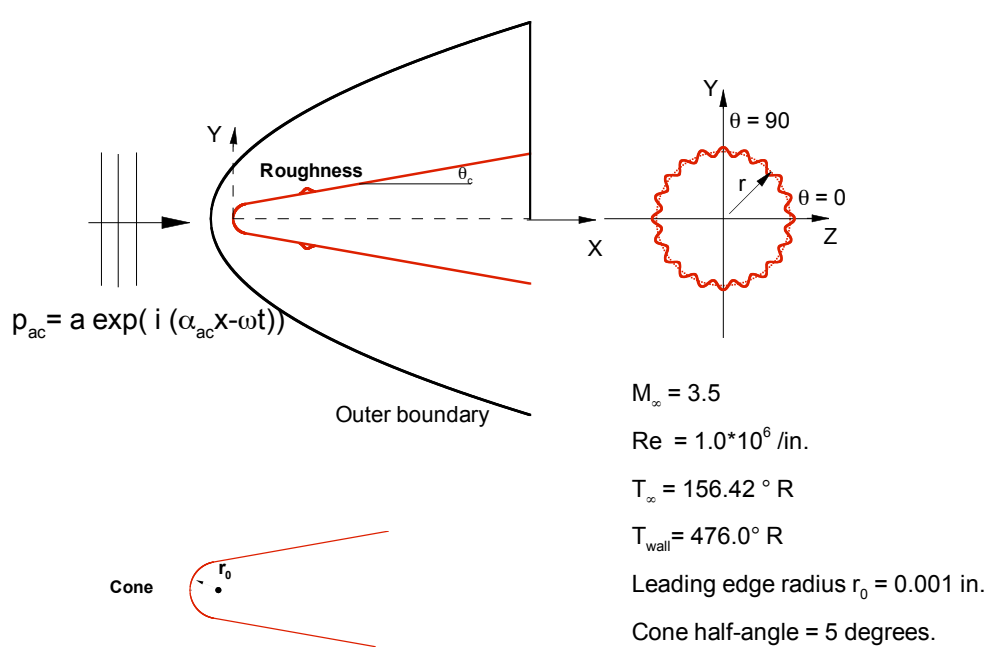

Figure 1. Schematic diagram of the computational model.

(a)

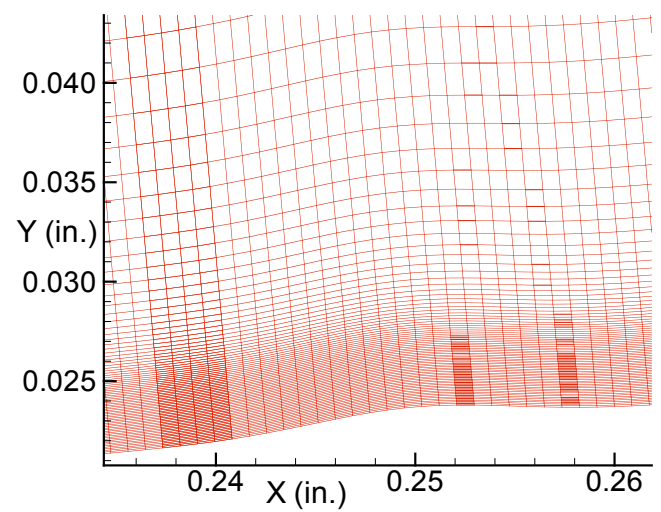

(b)

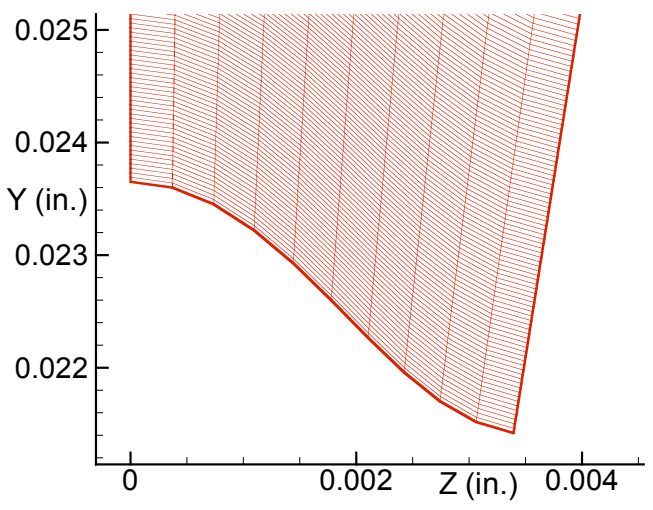

Figure 2. Grid distribution.

(a)

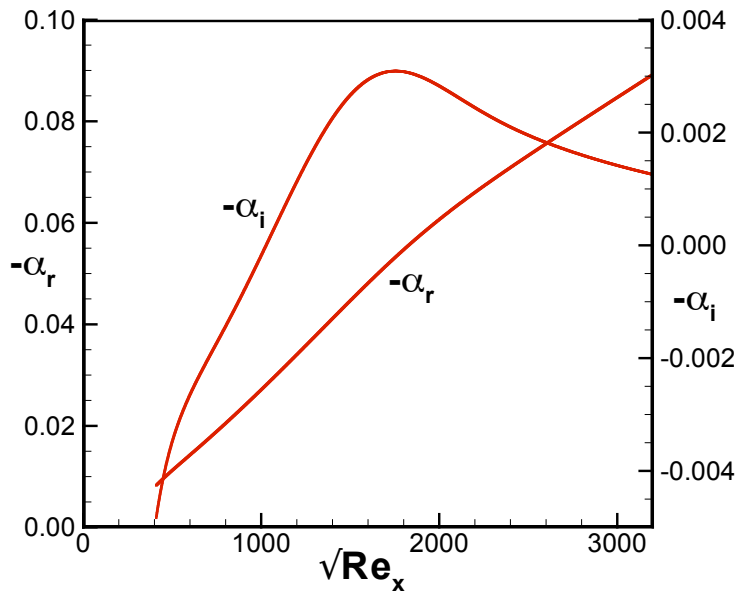

(b)

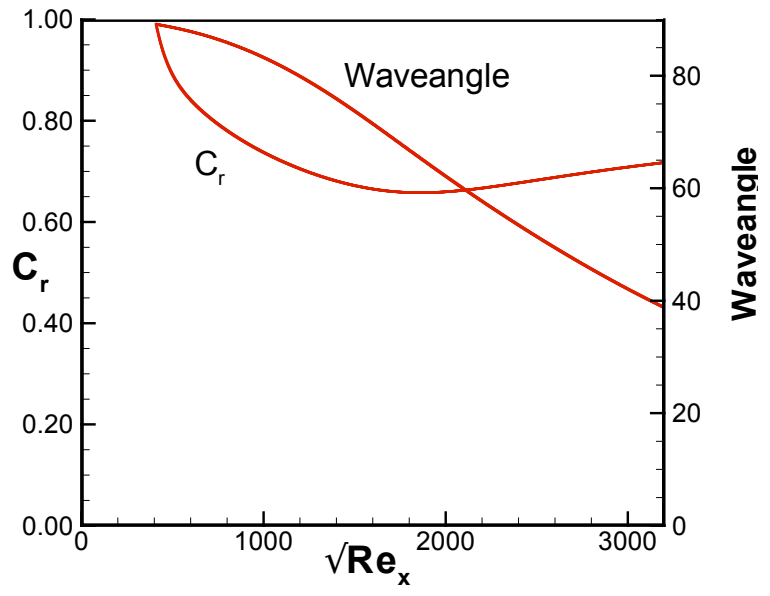

Figure 3. Wavenumber and growth rate (a) and phase speed and waveangle (b) for the boundary layer over a 5-deg cone. $M_{\infty}=3.5 . \mathrm{m}=20 \mathrm{~F}=2.0 * 10^{-5}$.

11 of 17

American Institute of Aeronautics and Astronautics 
(a)

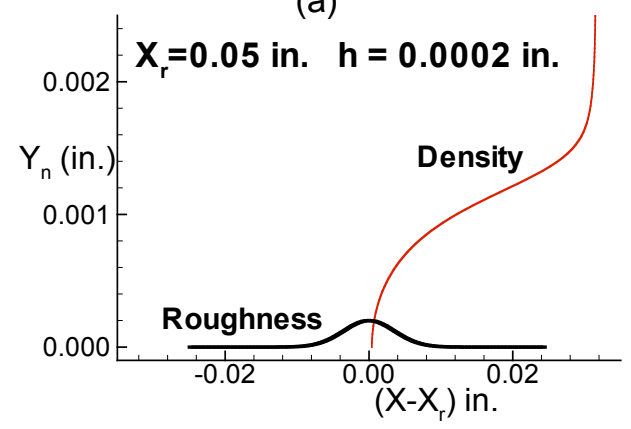

(c)

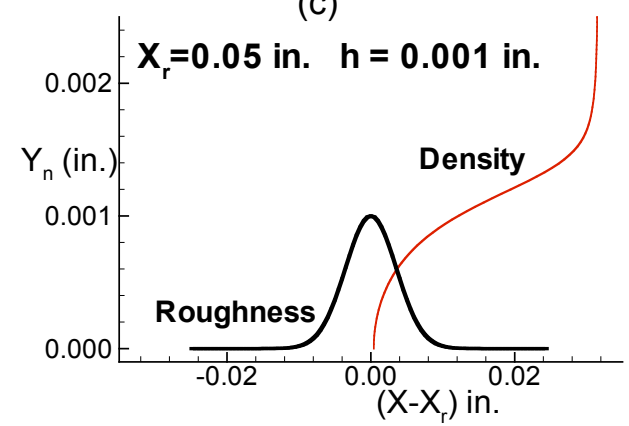

(e)

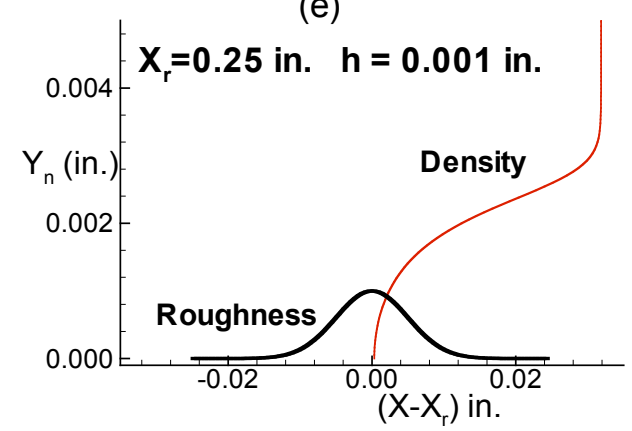

(b)

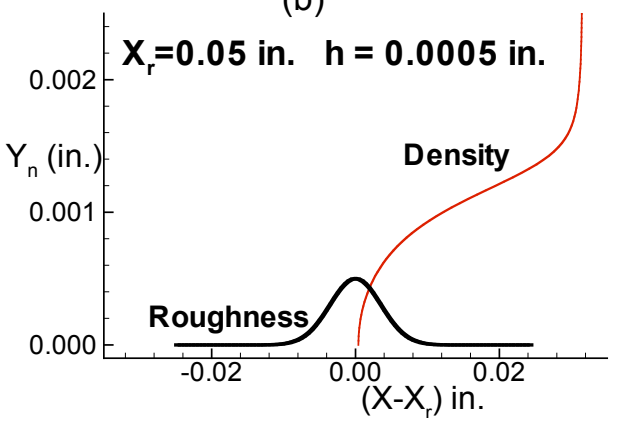

(d)

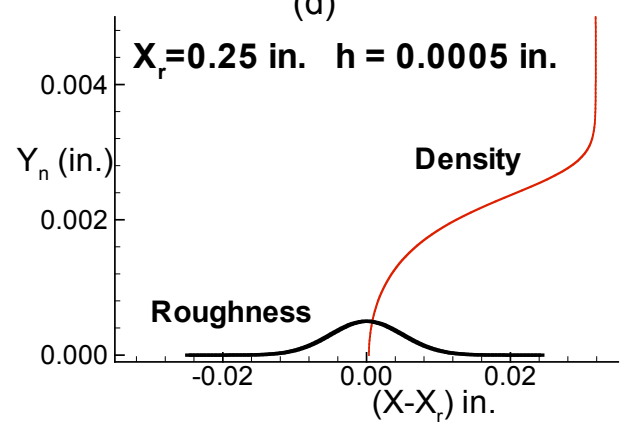

(f)

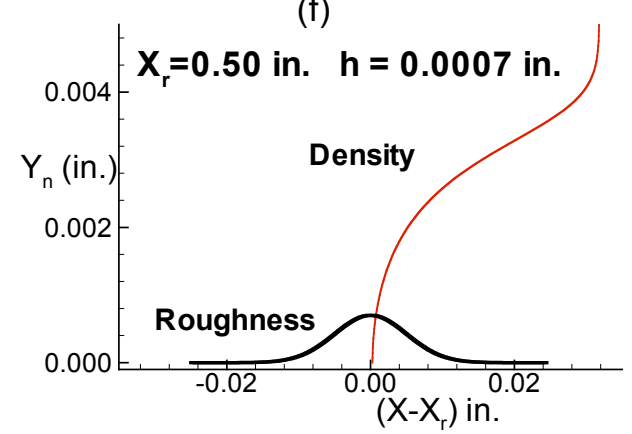

Figure 4. Density profile without the roughness and the roughness shape at different locations.
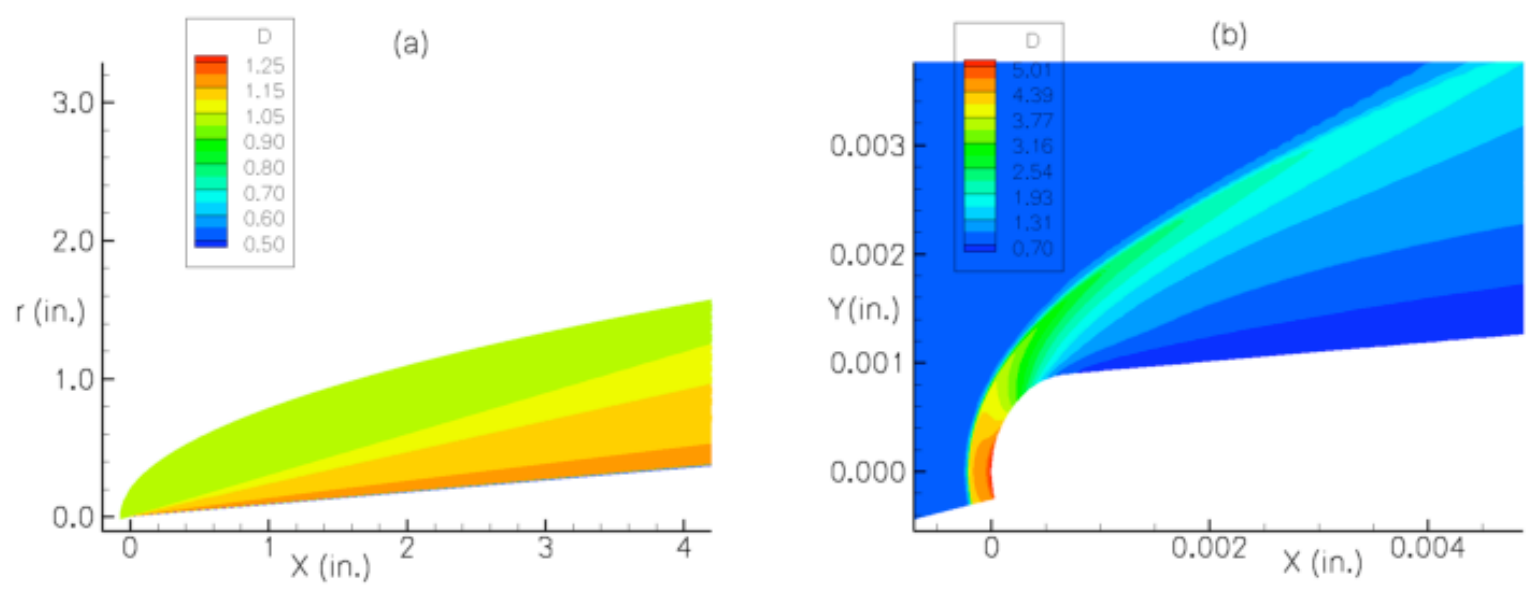

Figure 5. Contours of the density for flow over a 5-degree cone with a blunted leading edge at $M=3.5$. 
$38^{\text {th }}$ AIAA Fluid Dynamics Conference and Exhibit, June 23-26, 2008, Seattle, Washington

(a) $X_{f}=0.05$ in. $h=0.0005$ in.

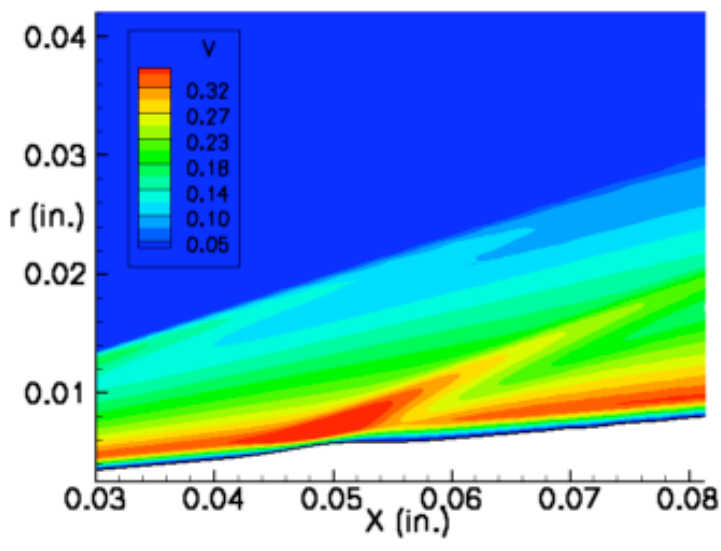

(c) $X_{t}=0.25$ in. $h=0.001$ in.

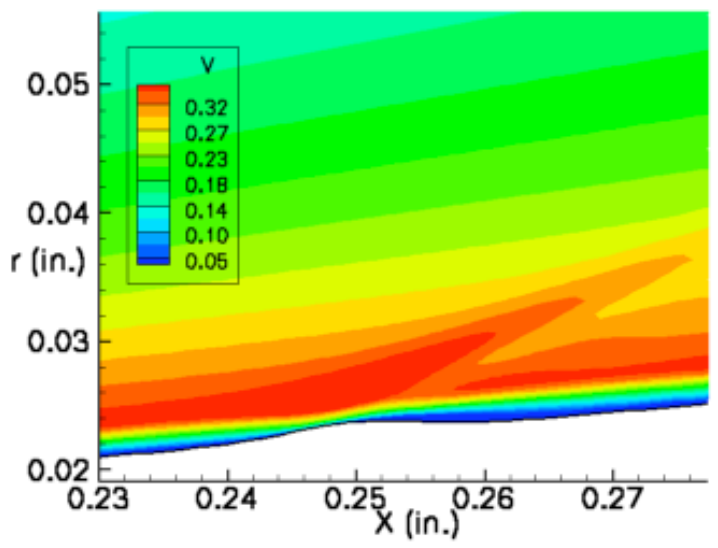

(b) $X_{t}=0.25$ in. $h=0.0005$ in.

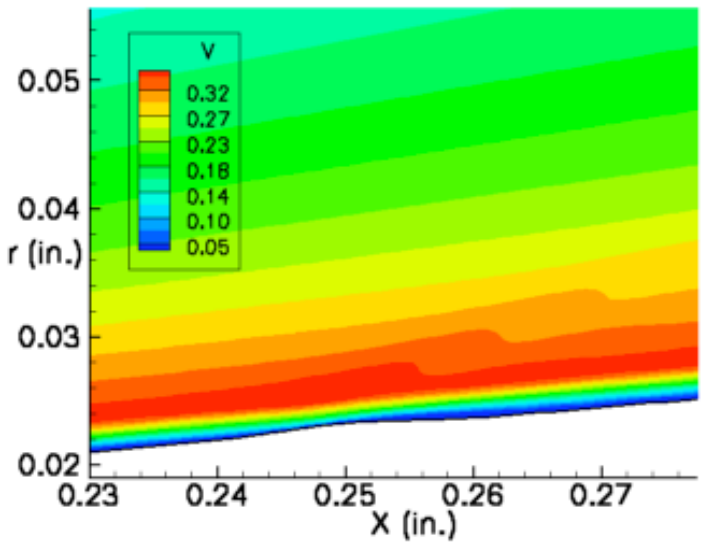

(d) $X_{t}=0.50$ in. $h=0.0007$ in.

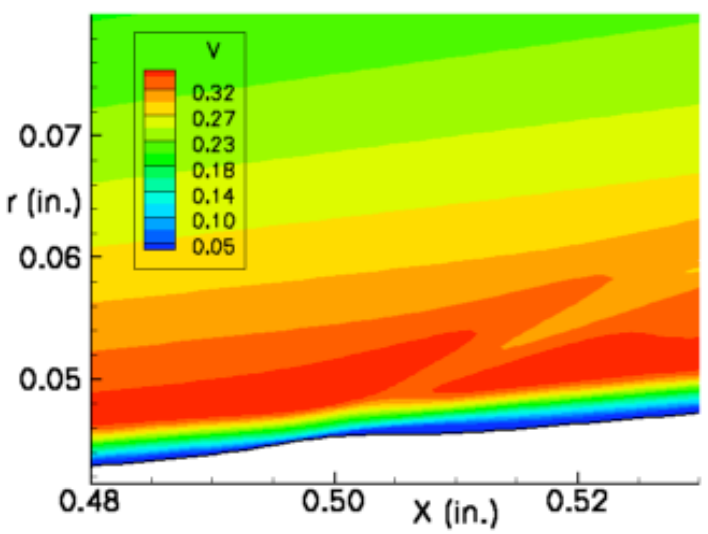

Figure 6. Contours of the radial velocity above the roughness $\mathbf{m}=\mathbf{2 0}$.

(a) $X_{t}=0.05$ in. $h=0.0005$ in.

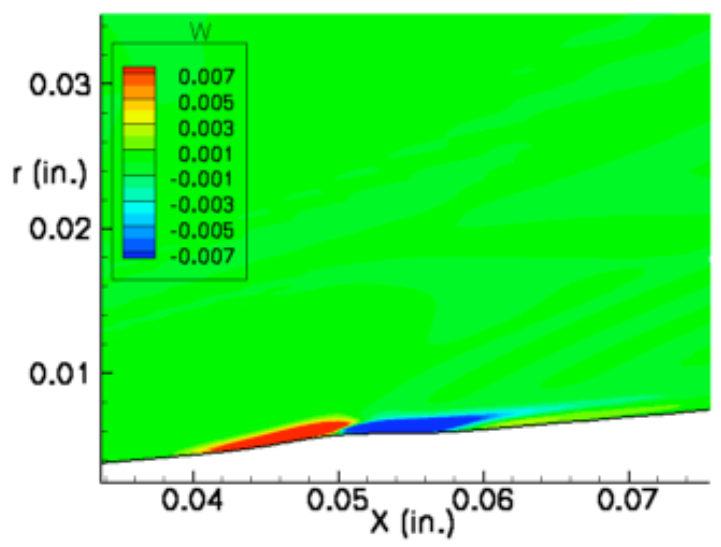

(b) $X_{f}=0.25$ in. $h=0.001$ in.

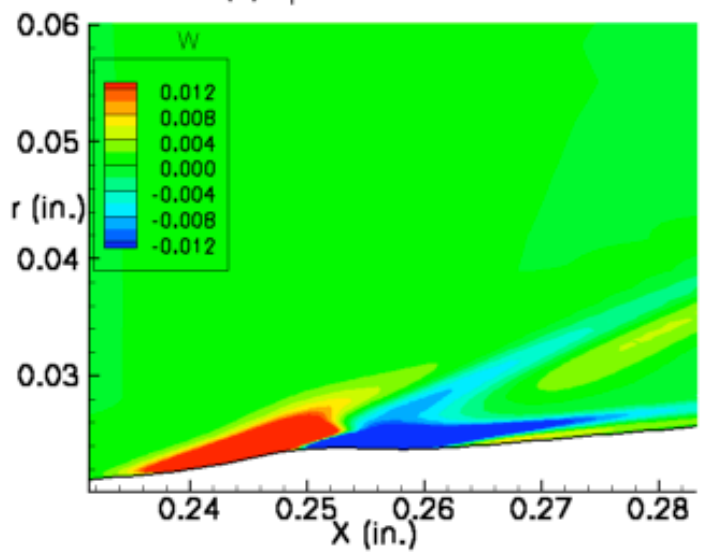

Figure 7. Contours of the azimuthal velocity above the roughness $\mathbf{m}=\mathbf{2 0}$. 
(a) $X=0.05$ in. $h=0.0002$ in.

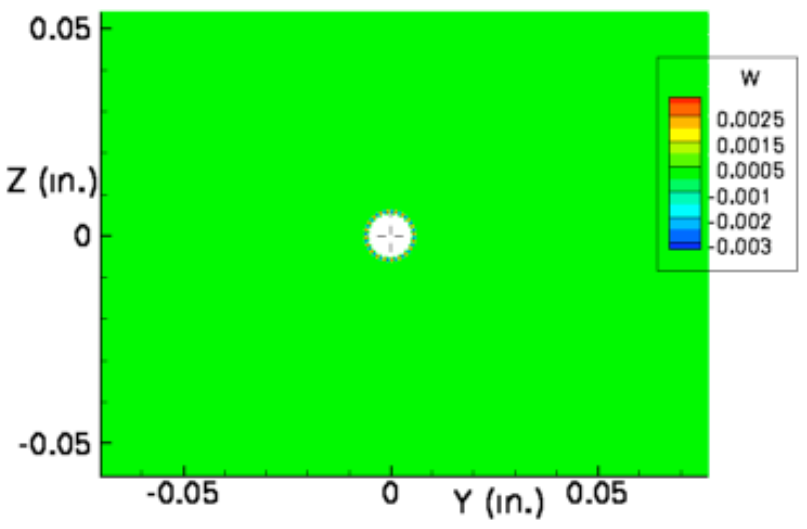

(c) $X=0.25$ in. $h=0.001$ in.

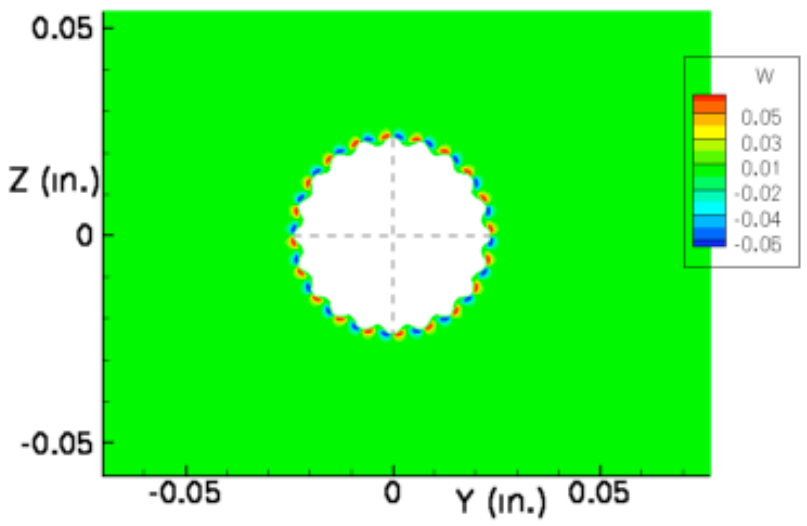

(b) $X=0.25$ in. $h=0.0005$ in.

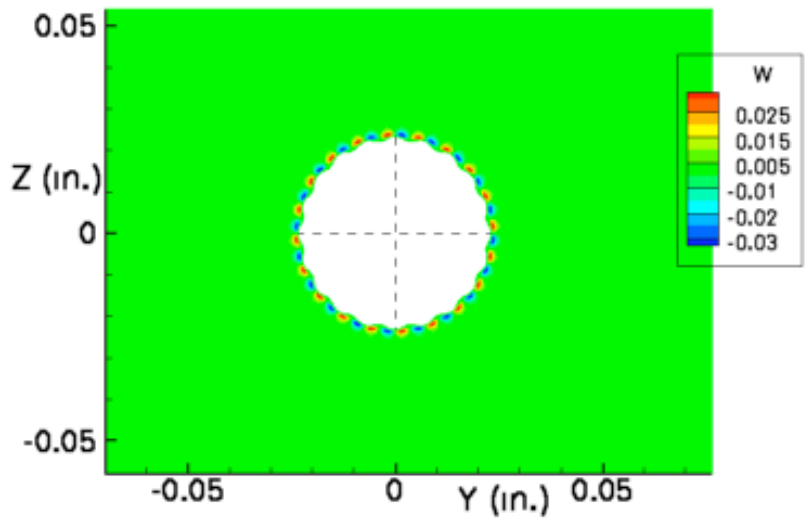

(d) $X=0.50$ in. $h=0.0007$ in.

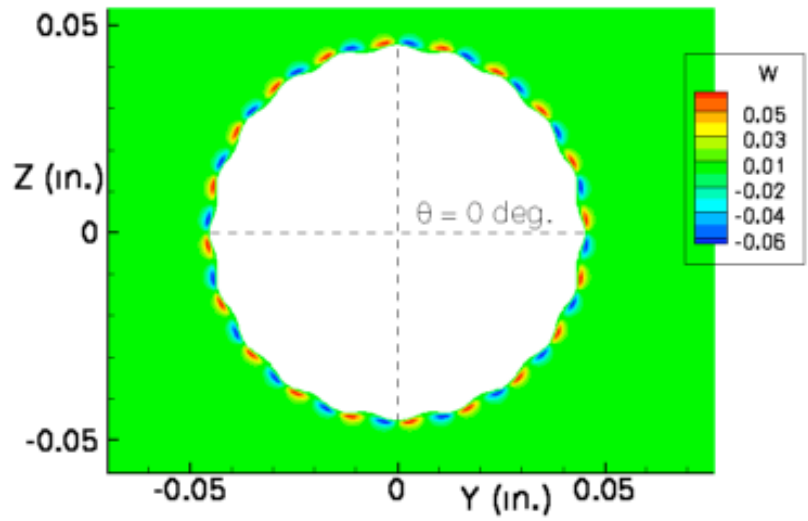

Figure 8. Contours of the azimuthal velocity above the roughness elements in the cross sectional plane (Y-Z). $\mathbf{m}=\mathbf{2 0}$.
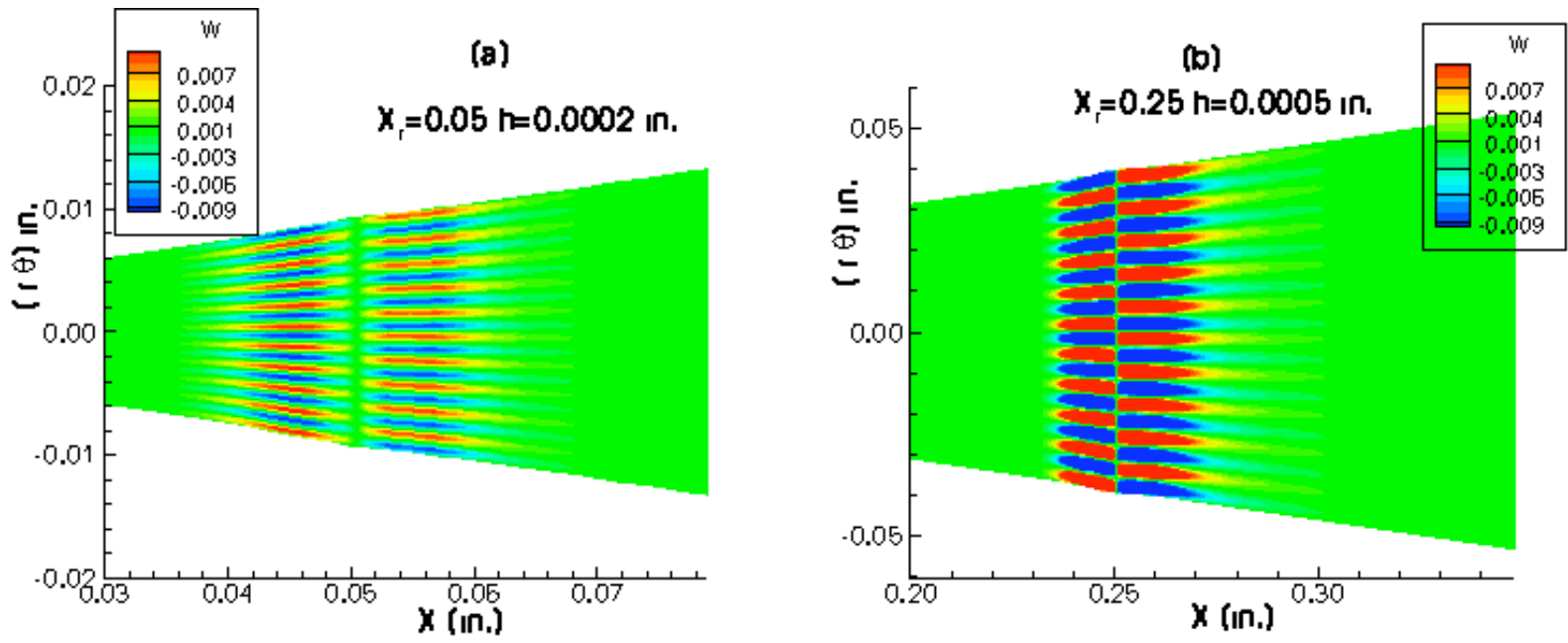

Figure 9. Contours of the azimuthal velocity in the $(X-r \theta)$ plane. $m=20$. 
(a)

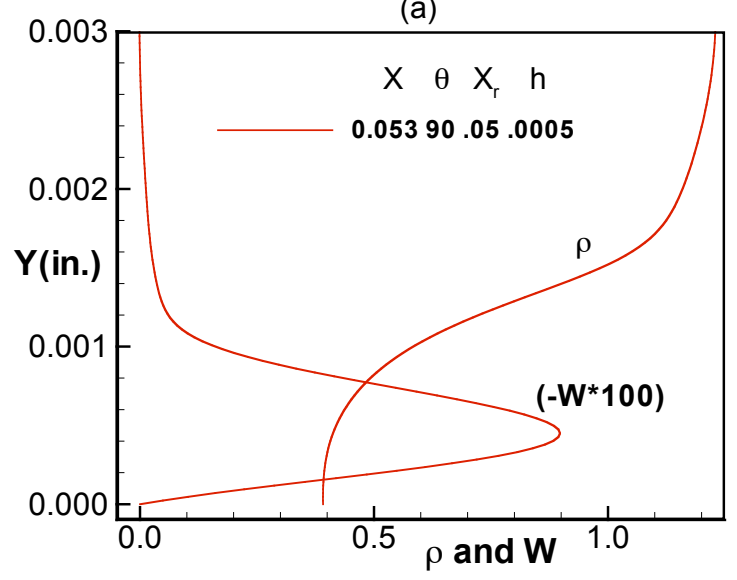

(b)

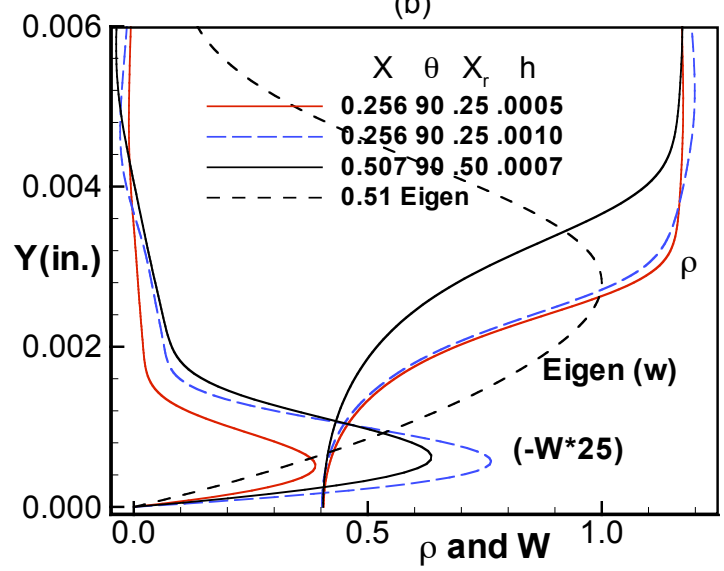

Figure 10. Density and the azimuthal velocity profiles above the roughness $\mathbf{m}=\mathbf{2 0}$.
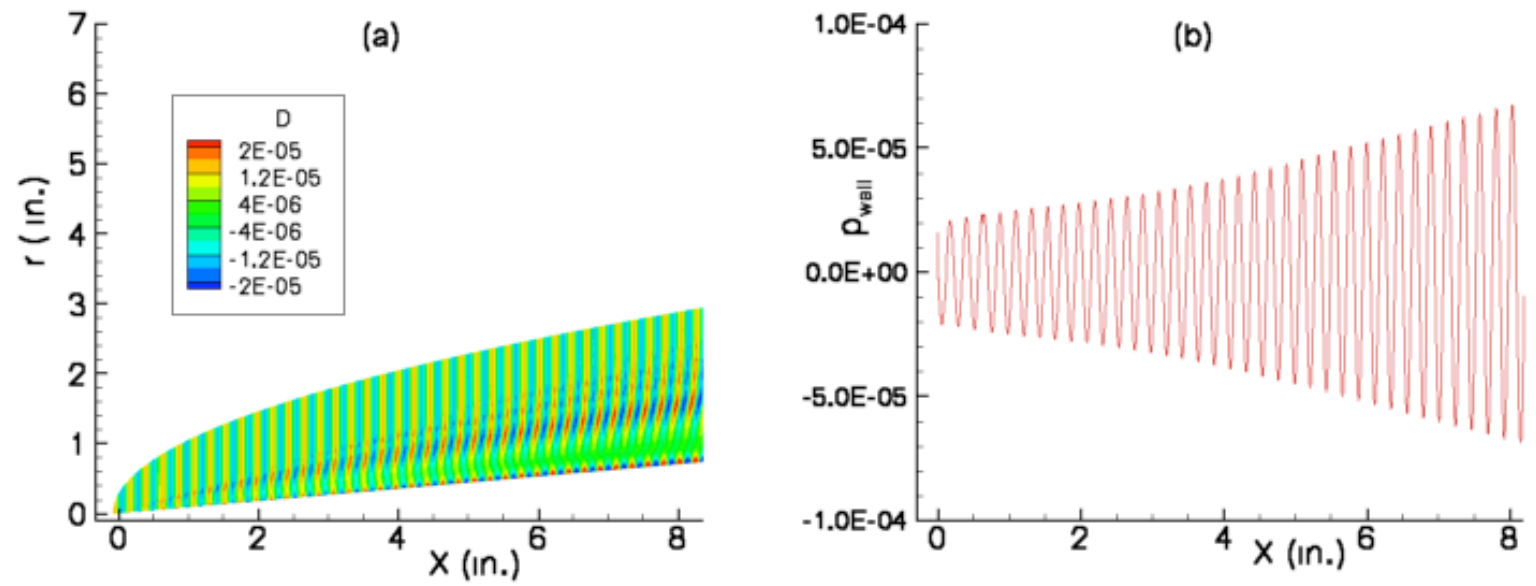

Figure 11. Contours of the density fluctuations (a) and the pressure fluctuations along the wall (b) due to $2 \mathrm{D}$ acoustic disturbances without the roughness. $F=2.0 * 10^{-5}, p_{a c} / p_{\infty}=1.0 * 10^{-5}$.

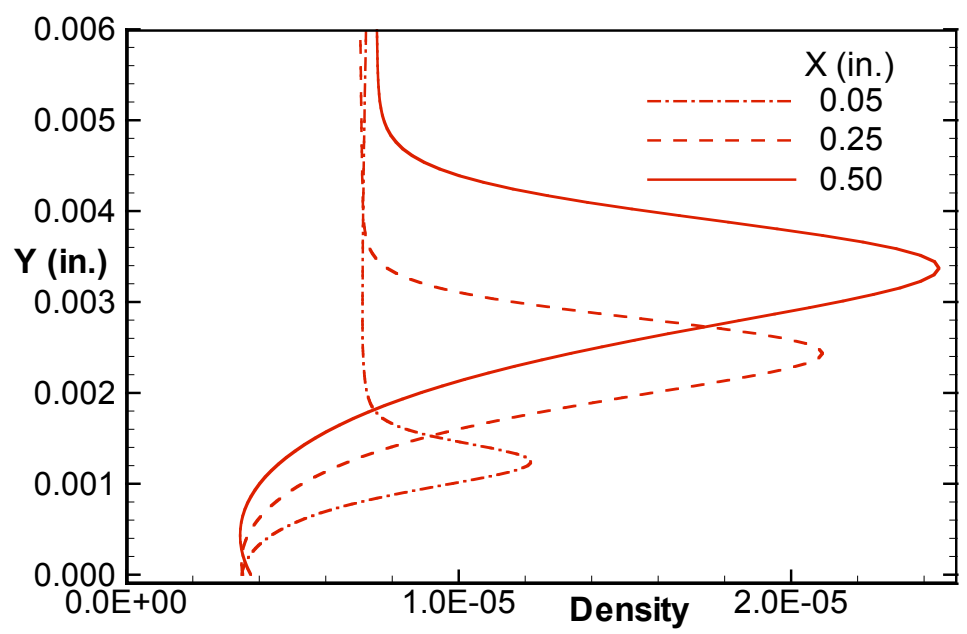

Figure 12. Amplitude of the density fluctuations inside the boundary layer at different axial locations due to $2 D$ acoustic disturbances without the roughness. $F=2.0 * 10^{-5}, p_{a c} / p_{\infty}=1.0 * 10^{-5}$.

15 of 17

American Institute of Aeronautics and Astronautics 
(a) Density perturbations, along $\theta=0$

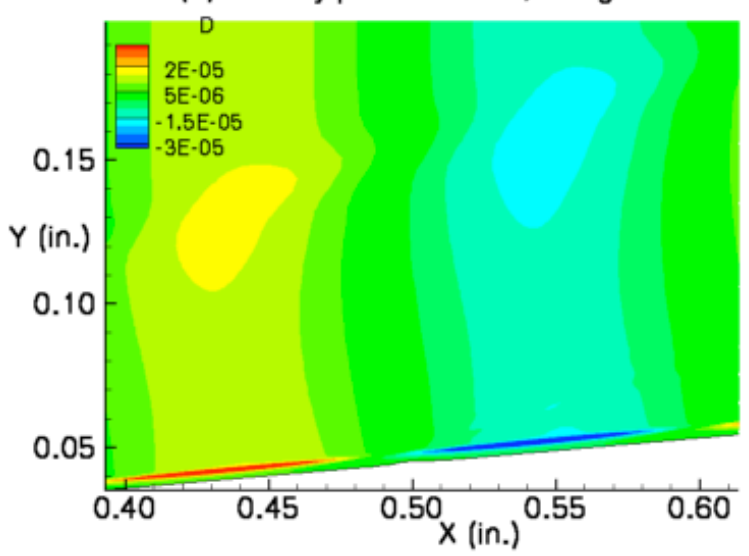

(b) Azimuthal velocity perturbations, along $\ominus=90 \mathrm{deg}$.

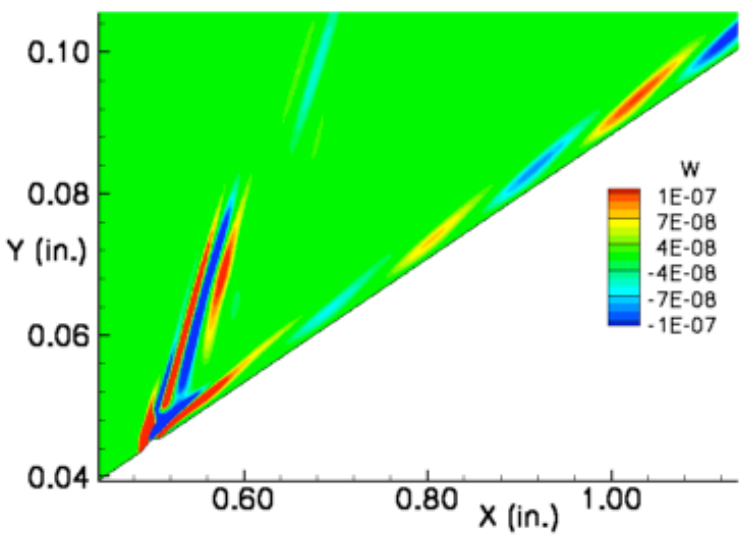

Figure 13. Contours of the density fluctuations (a) and the azimuthal velocity fluctuations (b) due to the interaction of $2 \mathrm{D}$ acoustic disturbances with the roughness. $X_{r}=0.50 \mathrm{in} ., \mathrm{h}=\mathbf{0 . 0 0 0 7} \mathrm{in}$., $\mathrm{m}=\mathbf{2 0}, \mathrm{F}=\mathbf{2 . 0} * 10^{-5}$, $\mathbf{p}_{\mathrm{ac}} / \mathbf{p}_{\infty}=1.0 * 10^{-5}$

(a)

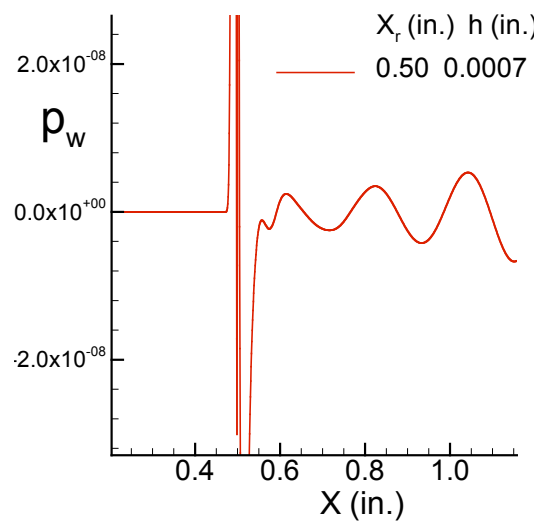

(b)

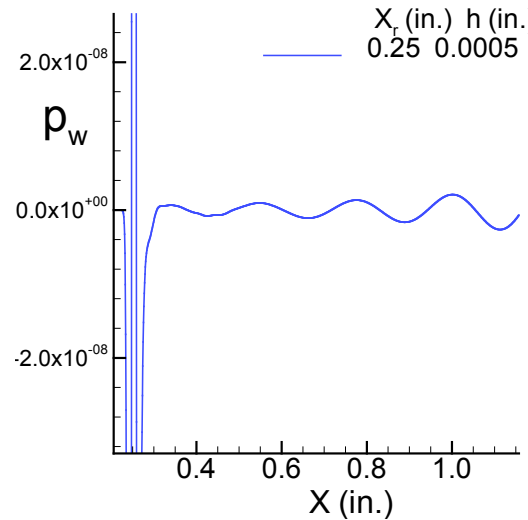

(c)

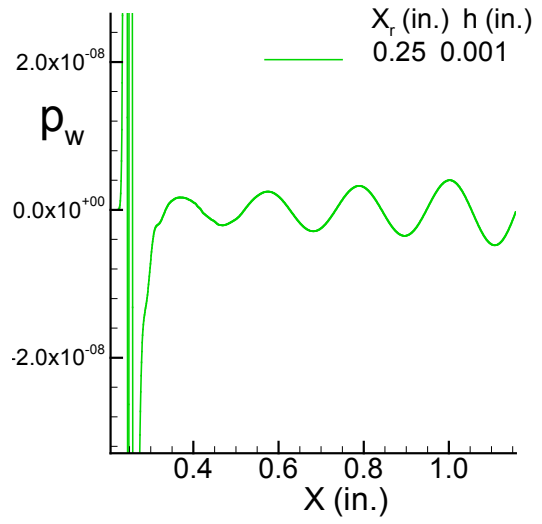

Figure 14. Pressure fluctuations of the instability waves along the wall close to the roughness due to the interaction of the acoustic waves with the roughness.

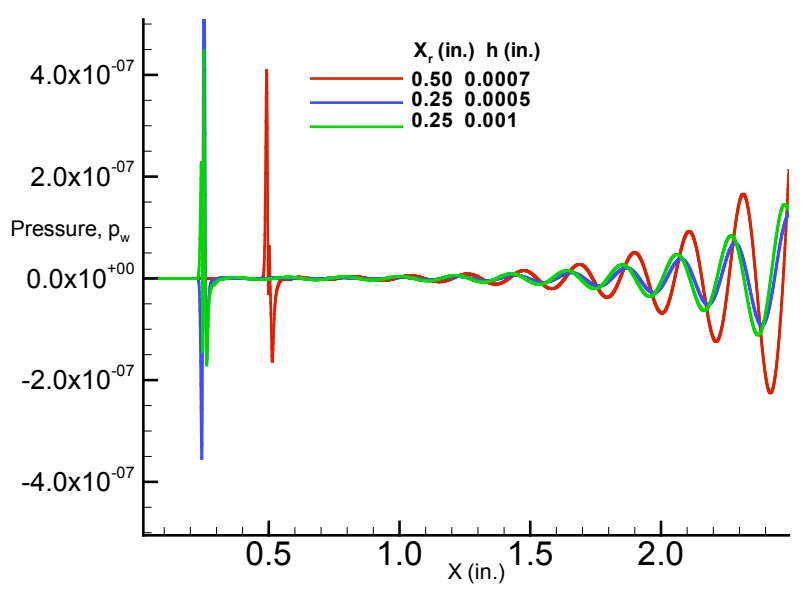

Figure 15. Growth of the pressure fluctuations of the instability waves along the wall due to the interaction of the acoustic disturbances with the roughness.

16 of 17

American Institute of Aeronautics and Astronautics 
(a)

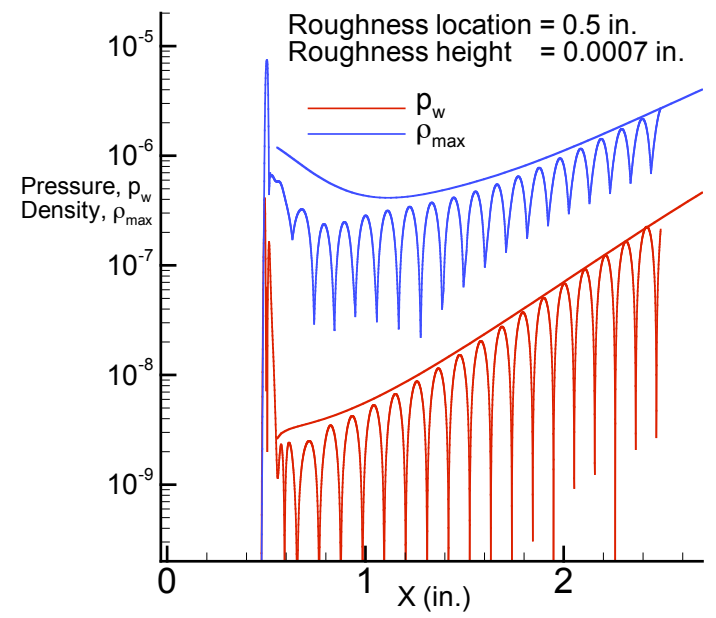

(b)

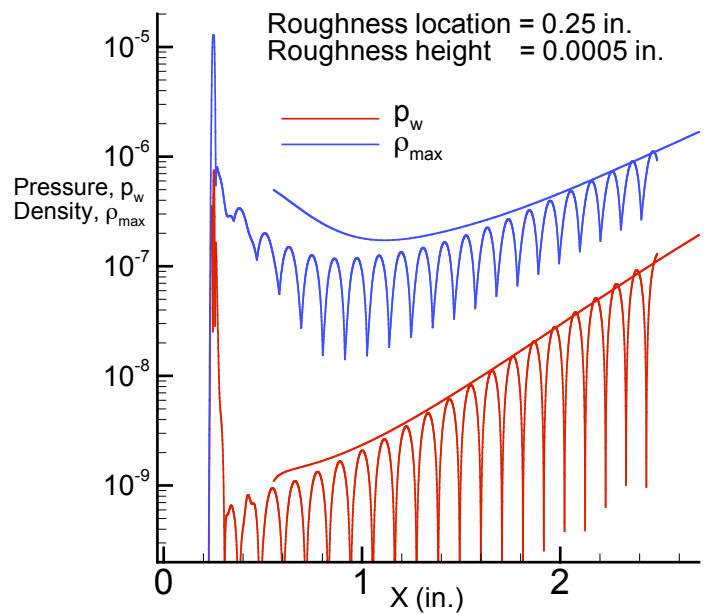

Figure 16. Pressure fluctuations and the maximum density fluctuations due to the interaction of the acoustic disturbances with the roughness.

(a)

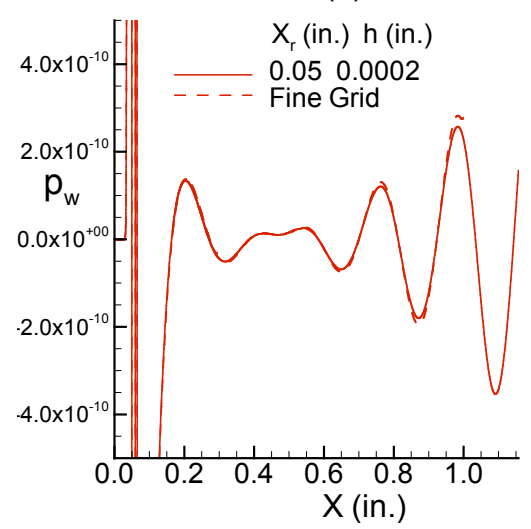

(b)

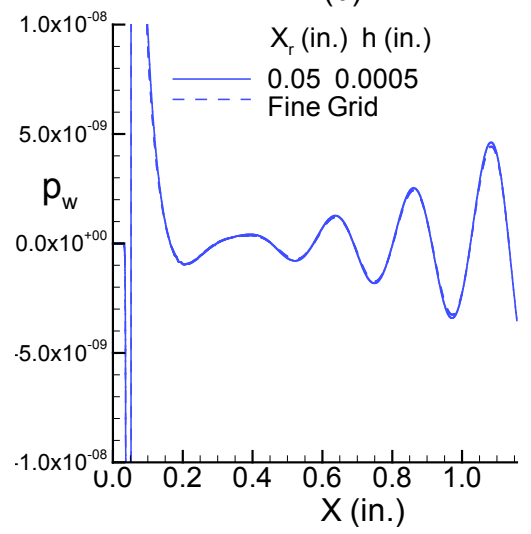

(c)

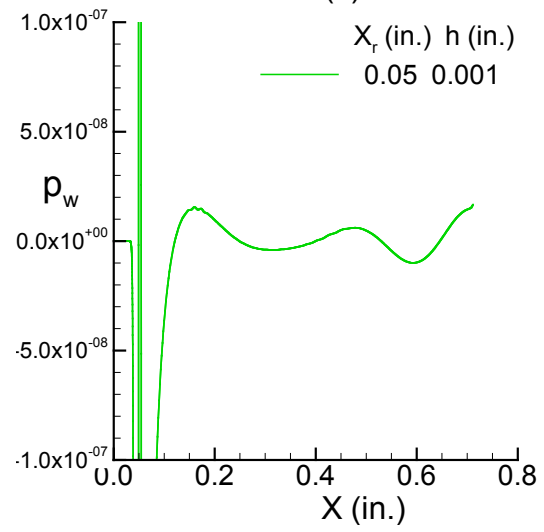

Figure 17. Pressure fluctuations of the instability waves along the wall due to the interaction of the acoustic disturbances with the roughness.
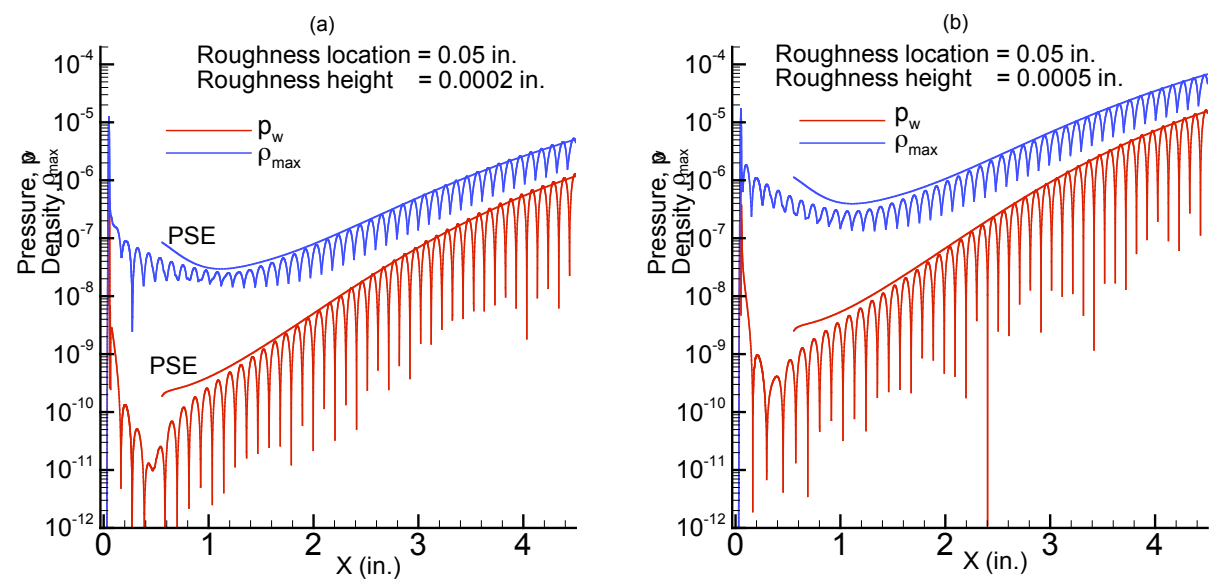

Figure 18. Pressure fluctuations and the maximum density fluctuations due to the interaction of the acoustic disturbances with the roughness.

17 of 17

American Institute of Aeronautics and Astronautics 